\title{
Dynamic modeling of the line throwing rocket with flight motion based on Kane's method
}

\author{
Lu Ming*, Gu Wenbin**, Liu Jianqing***, Wang Zhenxiong****, Xu Jinling***** \\ *Wuhan Ordnance N.C.O School, Wuhan 430075, china,E-mail: dosking001@163.com \\ **College of Field Engineering, PLA Univ. of Sci. \&Tech., Nanjing 210007, China, E-mail: guwenbin1@aliyun.com \\ ***College of Field Engineering, PLA Univ. of Sci. \&Tech., Nanjing 210007, China, E-mail: trainlimeng@163.com \\ ****College of Field Engineering, PLA Univ. of Sci. \&Tech., Nanjing 210007, China, E-mail: 249679787@qq.com \\ *****College of Field Engineering, PLA Univ. of Sci. \&Tech., Nanjing 210007, China, E-mail: 475216968@qq.com \\ cross $^{\text {ref }}$ http://dx.doi.org/10.5755/j01.mech.22.5.13376
}

\section{Introduction}

In recent years, affected by the extreme global climate change and seismic event, serious natural disasters occur frequently. However, a lot of rescue work was delayed because of blocking of the destroyed road and bridges as well as deep moat of the canyon, which poses a serious threat to the local residents' safety of life and property, and even causes serious disaster. Therefore, it's urgent to design an emergency rescue equipment to overcome obstacles of deep moat canyon and rapidly transport emergency relief supplies and people. The precision-guided line throwing rocket can send the rope to the opposite bank rapidly and precisely in complex geographical conditions, forming an air bridge in short time, thus, the staff and emergency relief supplies will be transported safely and efficiently. So it is of great significance to improve the efficiency of disaster relief and promote the national overall emergency rescue ability. And the research on flight dynamics of the precision-guided line throwing rocket is an important part of the entire development process.

The problem studied in this paper is put forward under this background. The working process can be described as follows: by the steel wire rope, the rocket was connected to one end of the rope of high strength which was neatly placed in the rope storage box. After launch, the rocket flies out with the power of the powder gas. At the same time, the rope is constantly pulled out from the rope storage box.

The rope in the flight is a flexible variable mass system, which has infinite degree of freedom and complex dynamics characteristics. Dynamic researches on the rope mainly focus on marine towing systems, space towing systems and high-altitude tethered drag systems. Many scholars carried out detailed researches on rope dynamics from different perspectives. McVey and Wolf developed the integration of axial and radial momentum equations, with which they predicted deployment and reefed ribbon parachutes [1]. Russell and Anderson used a two-degree-freedom lumped mass model to gain the understanding of the equilibrium and stability of a circularly towed cable [2]. Ablow and Schechter computed the motion of a towed cable with the finite difference approximation to the differential equations derived from basic dynamics [3]. Triantafyllou derived the static and linear zed dynamic governing equations along the local tangential and normal directions to study the dynamics of translating cables [4]. Niedzwecki and Thampi presented a general two-part analysis procedure for the investigation of snap load behavior of marine cable systems in regular seas [5]. Kamman and Huston presented an algorithm for modeling the dynamics of towed and tethered cable systems with fixed and varying lengths [6]. Driscoll used a one-dimensional finite-element lumped mass model to accurately reproduced eight snap loads and their non-linear characteristics occurred during the measurements for validation [7]. Buckham used the lumped mass approach to develop a mathematical model and computer simulation of an ROV tether operating in low-tension situations [8,9]. Quisenberry laid out a methodology for developing a numerical simulation of the aerial towed system [10]. It is obvious that the above researches mainly focus on the underwater towed systems [11-13], aerial towed systems [14-18] and parachute systems [19-21], while research on the flight of rocket with rope is less [22-24].

In the present paper, the finite segment model $[25,26]$ was used to deal with rope, and the dynamic modeling of the line throwing rocket was built based on Kane's method [27] was developed with its kinetic characteristics analyzed. The study has presented more accurate dynamic model on flight of the line throwing rocket, providing the theoretic model for research on disturbance of the rope, controlling as well as guidance on the line throwing rocket.

\section{The Kane's method}

\subsection{The basic concept}

1. Generalized coordinates, generalized velocity, partial velocity.

Suppose there is a system with $n$ particles in a selected frame of reference. The system has $n$ degrees of freedom and $n$ generalized coordinates. Assuming the position vector of the $i$-th particle is $r_{i}$. Then it can be written as:

$$
r_{i}=r_{i}\left(\begin{array}{lllll}
q_{1} & q_{2} & \ldots & q_{n} & t
\end{array}\right) \quad i=1,2, \ldots, n,
$$

where $q_{i}$ is the $i$-th generalized coordinate and $t$ is the time.

The velocity of the particle is defined to be the time derivative of the position vector. The velocity vector $v_{i}$ of the $i$-th particle can be written as:

$$
v_{i}=\frac{\partial r_{i}}{d t}=\sum_{j=1}^{l}\left(\frac{\partial r_{i}}{\partial q_{j}}\right) \dot{q}_{j}=\sum_{j=1}^{l} u_{i j} \dot{q}_{j} \quad i, j=1,2, \ldots, n,
$$

where $\dot{q}_{j}$ is defined to be the time derivative of $q_{j}$, it is the 
$j$-th generalized velocity. $u_{i j}=\frac{\partial r_{i}}{\partial q_{j}}=\frac{\partial v_{i}}{\partial \dot{q}_{j}}$ is the partial velocity of the $i$-th particle to the $j$-th generalized velocity.

2. Generalized active force and generalized inertial

force.

Generalized force is defined as the projection of the force along the side of the partial velocity. Assume that the mass of the $i$-th particle is $m_{i}$, the force acting on the point is $f_{i}$, its acceleration vector is $a_{i}$. Define $F_{j}=\sum_{i=1}^{n} f_{i} \times u_{i j}, F_{j}^{*}=\sum_{i=1}^{n} m_{i} a_{i} \times u_{i j}, F_{j}$ is the generalized active force, $F_{j}^{*}$ is the generalized inertial force.

3. Kane's equation.

According to Kane's method, the sum of generalized active force and generalized inertial force corresponding to the generalized velocity is zero. It is:

$$
F_{j}+F_{j}^{*}=0, \quad j=1,2, \ldots, n
$$

tem.

This is the Kane dynamics equation of particle sys-

If the system is multi-body system, the generalized active force and generalized inertia force are expressed as:

$$
\begin{aligned}
& F_{j}=\sum_{i=1}^{n} f_{i} \times u_{i j}+\sum_{i=1}^{n} t_{i} \times w_{i j}, \quad j=1,2, \ldots, n ; \\
& F_{j}^{*}=\sum_{i=1}^{n} f_{i}^{*} \times u_{i j}+\sum_{i=1}^{n} t_{i}^{*} \times w_{i j}, j=1,2, \ldots, n,
\end{aligned}
$$

where $f_{i}$ and $t_{i}$ are active force and moment of the rigid body, $f_{i}^{*}$ and $t_{i}^{*}$ are the inertial force and moment of the rigid body, $w_{i j}$ is its partial angular velocity.

\subsection{Advantages of the Kane's method}

Kane's method projects active force and inertial force along certain directions, which shows a clear geometric intuition. This approach not only has the advantage of Newtonian mechanics, but also has the advantages of analytical mechanics. There are no constraint forces in the differential equations, so the tedious analysis of the interactions between bodies are avoided; by generalized velocity to characterize motion of the system and developing the dynamic model of multi-body system through partial velocity and partial angular velocity, the number of equations is reduced and it is flexible to select independent variables; The introduction of generalized force and vector cross product instead of the complicated derivative operation makes it easy to write computer program for numerical calculation [28].

\section{Dynamic modeling of the line throwing rocket}

\subsection{Assumption}

Assume the whole system is located in a plane, and the motion is in the plane, it is a planar motion. The rocket is simplified as a mass point and the rope is broken into $n$ arbitrary segments in accordance with the finite segment method, wherein the length of each rope sections is $l_{i}$ and the last segment is variable-length and variable-mass segment, labels from the rocket pulled segment to the ground segment just follow $1,2,3, \ldots, n$. Without considerations on the elongation and bending of axial direction of the rope, assuming the mass of each rope segment mainly distributes on end of the segment further from the rocket, and different sections connected by a hinge, when the rope is pulled out and length of the last segment is changing till it fits the setting condition, a new rope segment $n+1$ will be pulled out.

\subsection{Kinematics analysis}

The coordinate system shown in Figure 1 is the inertial coordinate system. Define the launch point as the origin of coordinates. The $\mathrm{X}$ axis is along the rocket flight direction and is locally parallel to the 'ground'. The Y axis is vertical to the 'ground'. The derivation of the motion equations of the flexible cable and the rocket will be carried out in the inertial frame.

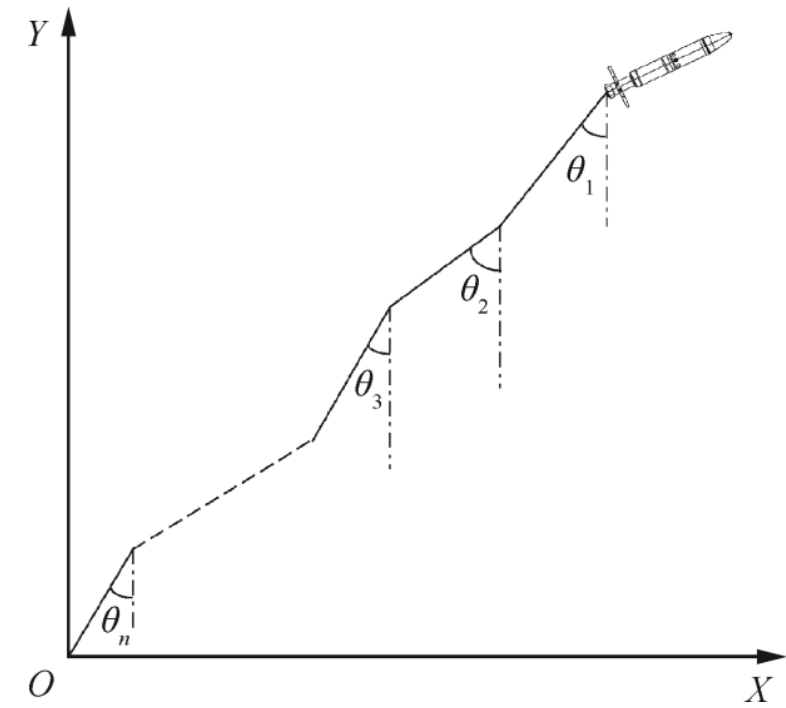

Fig. 1 Simplified model

\subsubsection{Position analysis}

At time $t$ the position of the rocket in the inertial frame are $\left[x_{0}(t) y_{0}(t)\right]$ ', and the angle between the $i$-th rope segment and the $\mathrm{Y}$ axis is $\theta_{i}(t)$. Select the rocket position $x_{0}(t), y_{0}(t)$, and define angles between each line segment and the $\mathrm{Y}$ axis $\theta_{i}(t)$ as the generalized coordinates. There are totally $n+2$ generalized coordinates. Then the position of each rope segment is:

$$
\left[\begin{array}{l}
x_{i}(t) \\
y_{i}(t)
\end{array}\right]=\left[\begin{array}{l}
x_{i-1}(t)-l_{i} \sin \theta_{i}(t) \\
y_{i-1}(t)-l_{i} \cos \theta_{i}(t)
\end{array}\right], \quad i=1,2, \ldots, n .
$$

\subsubsection{Velocity analysis}

The velocity is produced by derivation of the position of the rocket and each rope segment. Define $\dot{\theta}_{i}(t)=\omega_{i}(t)$, so velocity of the rocket is $\left[\dot{x}_{0}(t) \dot{y}_{0}(t)\right]$, and velocity of the intensive mass point on each rope segment is: 
$\left[\begin{array}{c}\dot{x}_{i}(t) \\ \dot{y}_{i}(t)\end{array}\right]=\left[\begin{array}{c}\dot{x}_{i-1}(t)-l_{i} \cos \theta_{i}(t) \omega_{1}(t) \\ \dot{y}_{i-1}(t)+l_{i} \sin \theta_{i}(t) \omega_{1}(t)\end{array}\right], i=1,2, \ldots, n$

\subsubsection{Partial velocity analysis}

By the Kane's method, we can get the partial velocity of each rope segment to generalized velocity as Table 1 .

That is

$$
u_{i j}= \begin{cases}{\left[\begin{array}{l}
0 \\
0
\end{array}\right]} & i<j, j \leq n ; \\
{\left[\begin{array}{l}
1 \\
0
\end{array}\right]} & j=n+1 ; \\
{\left[\begin{array}{l}
0 \\
1
\end{array}\right]} & j=n+2 ; \\
{\left[\begin{array}{l}
-l_{i} \cos \theta_{i}(t) \\
l_{i} \sin \theta_{i}(t)
\end{array}\right]} & i \geq j .\end{cases}
$$

By derivation of the partial velocity derivative of the partial velocity can be obtained as is shown in Table 2 .

That is

$$
\dot{u}_{i j}= \begin{cases}{\left[\begin{array}{l}
0 \\
0
\end{array}\right]} & i<j ; \\
{\left[\begin{array}{ll}
l_{i} \sin \theta_{i}(t) \omega_{i}(t) \\
l_{i} \cos \theta_{i}(t) \omega_{i}(t)
\end{array}\right]} & i \geq j .\end{cases}
$$

\subsubsection{Acceleration analysis}

Derivation of velocity of the rocket and each rope segment produces their acceleration, and acceleration of the rocket and each rope segment are

$$
a_{i}=\sum_{j=0}^{n+1} u_{i j} \ddot{q}_{j}+\dot{u}_{i j} \dot{q}_{j}, \quad i=0,1,2, \ldots, n,
$$

\begin{tabular}{|c|c|c|c|c|c|c|}
\hline & $\omega_{1}(t)$ & $\omega_{2}(t)$ & $\ldots$ & $\omega_{n}(t)$ & $\dot{x}_{0}(t)$ & $\dot{y}_{0}(t)$ \\
\hline 0 & {$\left[\begin{array}{l}0 \\
0\end{array}\right]$} & {$\left[\begin{array}{l}0 \\
0\end{array}\right]$} & $\cdots$ & {$\left[\begin{array}{l}0 \\
0\end{array}\right]$} & {$\left[\begin{array}{l}1 \\
0\end{array}\right]$} & {$\left[\begin{array}{l}0 \\
1\end{array}\right]$} \\
\hline 1 & {$\left[\begin{array}{l}-l_{1} \cos \theta_{1}(t) \\
l_{1} \sin \theta_{1}(t)\end{array}\right]$} & {$\left[\begin{array}{l}0 \\
0\end{array}\right]$} & $\cdots$ & {$\left[\begin{array}{l}0 \\
0\end{array}\right]$} & {$\left[\begin{array}{l}1 \\
0\end{array}\right]$} & {$\left[\begin{array}{l}0 \\
1\end{array}\right]$} \\
\hline 2 & {$\left[\begin{array}{l}-l_{1} \cos \theta_{1}(t) \\
l_{1} \sin \theta_{1}(t)\end{array}\right]$} & {$\left[\begin{array}{l}-l_{2} \cos \theta_{2}(t) \\
l_{2} \sin \theta_{2}(t)\end{array}\right]$} & $\cdots$ & {$\left[\begin{array}{l}0 \\
0\end{array}\right]$} & {$\left[\begin{array}{l}1 \\
0\end{array}\right]$} & {$\left[\begin{array}{l}0 \\
1\end{array}\right]$} \\
\hline$\ldots$ & $\ldots$ & $\ldots$ & $\ldots$ & $\ldots$ & $\ldots$ & $\ldots$ \\
\hline $\mathrm{n}$ & {$\left[\begin{array}{l}-l_{1} \cos \theta_{1}(t) \\
l_{1} \sin \theta_{1}(t)\end{array}\right]$} & {$\left[\begin{array}{l}-l_{2} \cos \theta_{2}(t) \\
l_{2} \sin \theta_{2}(t)\end{array}\right]$} & $\cdots$ & {$\left[\begin{array}{l}-l_{n} \cos \theta_{n}(t) \\
l_{n} \sin \theta_{n}(t)\end{array}\right]$} & {$\left[\begin{array}{l}1 \\
0\end{array}\right]$} & {$\left[\begin{array}{l}0 \\
1\end{array}\right]$} \\
\hline
\end{tabular}

where $\dot{q}_{j}$ is the $j^{\text {th }}$ generalized velocity, $\ddot{q}_{j}$ is derivative of

\begin{tabular}{|c|c|c|c|c|c|c|}
\hline & $\omega_{1}(t)$ & $\omega_{2}(t)$ & $\ldots$ & $\omega_{n}(t)$ & $\dot{x}_{0}(t)$ & $\dot{y}_{0}(t)$ \\
\hline 0 & {$\left[\begin{array}{l}0 \\
0\end{array}\right]$} & {$\left[\begin{array}{l}0 \\
0\end{array}\right]$} & $\cdots$ & {$\left[\begin{array}{l}0 \\
0\end{array}\right]$} & {$\left[\begin{array}{l}0 \\
0\end{array}\right]$} & {$\left[\begin{array}{l}0 \\
0\end{array}\right]$} \\
\hline 1 & {$\left[\begin{array}{l}l_{1} \sin \theta_{1}(t) \omega_{1}(t) \\
l_{1} \cos \theta_{1}(t) \omega_{1}(t)\end{array}\right]$} & {$\left[\begin{array}{l}0 \\
0\end{array}\right]$} & $\ldots$ & {$\left[\begin{array}{l}0 \\
0\end{array}\right]$} & $\left.\begin{array}{l}0 \\
0\end{array}\right]$ & {$\left[\begin{array}{l}0 \\
0\end{array}\right]$} \\
\hline 2 & {$\left[\begin{array}{l}l_{1} \sin \theta_{1}(t) \omega_{1}(t) \\
l_{1} \cos \theta_{1}(t) \omega_{1}(t)\end{array}\right]$} & {$\left[\begin{array}{l}l_{2} \sin \theta_{2}(t) \omega_{2}(t) \\
l_{2} \cos \theta_{2}(t) \omega_{2}(t)\end{array}\right]$} & $\cdots$ & {$\left[\begin{array}{l}0 \\
0\end{array}\right]$} & {$\left[\begin{array}{l}0 \\
0\end{array}\right]$} & {$\left[\begin{array}{l}0 \\
0\end{array}\right]$} \\
\hline$\ldots$ & $\ldots$ & $\ldots$ & $\ldots$ & $\ldots$ & $\ldots$ & $\ldots$ \\
\hline $\mathrm{n}$ & {$\left[\begin{array}{l}l_{1} \sin \theta_{1}(t) \omega_{1}(t) \\
l_{1} \cos \theta_{1}(t) \omega_{1}(t)\end{array}\right]$} & {$\left[\begin{array}{l}l_{2} \sin \theta_{2}(t) \omega_{2}(t) \\
l_{2} \cos \theta_{2}(t) \omega_{2}(t)\end{array}\right]$} & $\cdots$ & {$\left[\begin{array}{l}l_{n} \sin \theta_{n}(t) \omega_{n}(t) \\
l_{n} \cos \theta_{n}(t) \omega_{n}(t)\end{array}\right]$} & {$\left[\begin{array}{l}0 \\
0\end{array}\right]$} & {$\left[\begin{array}{l}0 \\
0\end{array}\right]$} \\
\hline
\end{tabular}
the $j^{\text {th }}$ generalized velocity.

Table 1

Partial velocity

Table 2

The derivative of the partial velocity 


\subsection{Dynamic analysis}

\subsubsection{Generalized active force}

(1) Gravity fg. Quality of the rocket is $m_{0}$ and gravity of the rocket can be obtained as:

$$
f g_{0}=\left[\begin{array}{l}
0 \\
m_{0} g
\end{array}\right],
$$

the linear density of the rope is $\rho$, the quality of each rope segment is $m_{i}=\rho l_{i}$, and gravity of each rope segment can be obtained as:

$$
f g_{i}=\left[\begin{array}{l}
0 \\
m_{i} g
\end{array}\right], \quad i=1,2, \ldots, n .
$$

(2) Aerodynamic forces $f d$. The rocket will be effected by aerodynamic forces when it flying in the air. Experimental results show that: the aerodynamic forces acting on the rocket is proportional to the dynamic pressure of the flow and the characteristics area of the rocket [29]. Assuming that the rocket axis coincides with the velocity vector, then the aerodynamic force acting on rocket is along the shaft backwards, and it is air resistance:

$$
f d_{0}=\left[\begin{array}{c}
c_{0} q_{0 x} S_{0} \\
c_{0} q_{0 y} S_{0}
\end{array}\right],
$$

where $c_{0}$ is drag coefficient of the rocket, $S_{0}$ is the rocket characteristic area, $q_{0 x}=\frac{1}{2} \rho_{a} V_{x}^{2}, q_{0 y}=\frac{1}{2} \rho_{a} V_{y}^{2}$ are dynamic pressures, $\rho_{a}$ is the air density situated at the height of the rocket, $V_{x}, V_{y}$ represent velocities in the $\mathrm{X}$ - and Y-axis direction.

Because the soft fabric has breathability, it is very difficult to accurately calculate drag force of the rope. Considering the engineering requirements, we can get the air resistance acting on each rope segment as the air resistance acting on the rocket:

$$
f d_{i}=\left[\begin{array}{c}
c_{i} q_{i x} S_{i} \\
c_{i} q_{i y} S_{i}
\end{array}\right] \quad i=1,2, \ldots, n,
$$

where $c_{i}$ is the drag coefficient of the $i$-th rope segment, $S_{i}$ is characteristic area of the $i$-th rope segment, $q_{i x}$ and $q_{i y}$ are dynamic pressures of the $i$-th segment in the $\mathrm{X}$ - and Y-axis direction.

(3) Thrust fi. After launch, the engine starts to work, gunpowder gas combusts, the combustion products emits from the nozzle thus promote the rocket to fly forward. This is the driving force to promote the rocket, the force would be acting on the rocket until the engine stop working, and it is thrust. Assuming that the thrust I is along the axis of the rocket, it can be written as:

$$
f i_{i}= \begin{cases}{\left[\begin{array}{l}
I_{x} \\
I_{y}
\end{array}\right]} & i=0 ; \\
{\left[\begin{array}{l}
0 \\
0
\end{array}\right]} & i=1,2, \ldots, n,\end{cases}
$$

where $I_{x}, I_{y}$ are thrust in the X-and Y-axis direction.

(4) The Forces Acting on the Last Segment $f t$. When the rope is pulled out, the forces acting on the last segment are very complex. According to Wolf's pulling model of straight line [30], assuming the forces acting on the last segment is $T$ :

$$
f t_{i}= \begin{cases}{\left[\begin{array}{l}
0 \\
0
\end{array}\right]} & i=0,1,2, \ldots, n-1 ; \\
{\left[\begin{array}{l}
T_{x} \\
T_{y}
\end{array}\right]} & i=n,\end{cases}
$$

where $T_{x}, T_{y}$ are forces acting on the last segment in the $\mathrm{X}$ and $\mathrm{Y}$-axis direction.

(5) Combination of Active Forces $f$. The combination of active forces acting on the rocket and each rope segment can be written as:

$$
f z_{i}=f g_{i}+f d_{i}+f i_{i}+f t_{i} \quad i=0,1,2, \ldots, n,
$$

its matrix formulation is:

$$
f z=f g+f d+f i+f t .
$$

(6) Generalized Active Force Fl. According to the Kane's method, generalized active force of the $j$-th generalized coordinates is:

$$
F l_{j}=\sum_{i=1}^{n} f z_{i} u_{i j}, \quad j=1,2, \ldots, n+2 .
$$
is:

The generalized active force of the whole system

$$
F l=u^{\prime} f z
$$

\subsubsection{Generalized Inertial Force $F l^{*}$}

According to the Kane's method, The generalized inertial force of the $j$-th generalized coordinates is:

$$
\left.\begin{array}{l}
F l_{j}^{*}=\sum_{i=1}^{n}-m_{i} a_{i} u_{i j}=\sum_{i=1}^{n}-m_{i}\left(u_{i j} \ddot{q}_{j}+\dot{u}_{i j} \dot{q}_{j}\right) u_{i j} ; \\
j=1,2, \ldots, n+2 .
\end{array}\right\}
$$

Generalized inertial force of the whole system is:

$$
F l^{*}=-u^{\prime} M u \ddot{q}-u^{\prime} M \dot{u} \dot{q},
$$

where $u, \dot{q}, \ddot{q}$ are matrixes formulations of $u_{i j}, \dot{q}_{j}, \ddot{q}_{j}, u^{\prime}$ is the transpose matrix of $u, M=\operatorname{diag}\left[m_{0}, m_{1}, \ldots, m_{n}\right]$.

\subsection{Dynamic equation}

According to the Kane equation

$$
F l+F l^{*}=0 .
$$

Put Eq. (22) into Eq. (23), we have: 
$F l-u^{\prime} M u \ddot{q}-u^{\prime} M \dot{u} \dot{q}=0$.

By transposition:

$$
u^{\prime} M u \ddot{q}=F l-u^{\prime} M \dot{u} \dot{q} \text {. }
$$

Formula (25) can be written as:

$$
A \ddot{q}=f l,
$$

where $A=u^{\prime} M u, f l=F l-u^{\prime} M \dot{u} \dot{q}$.

Formula (26) includes $n+2$ equations and totally $n+2$ variables. Then the kinematic parameters of the rocket and rope sections can be obtained.

\subsection{The calculation program}

The calculation program was shown in Fig. 2.

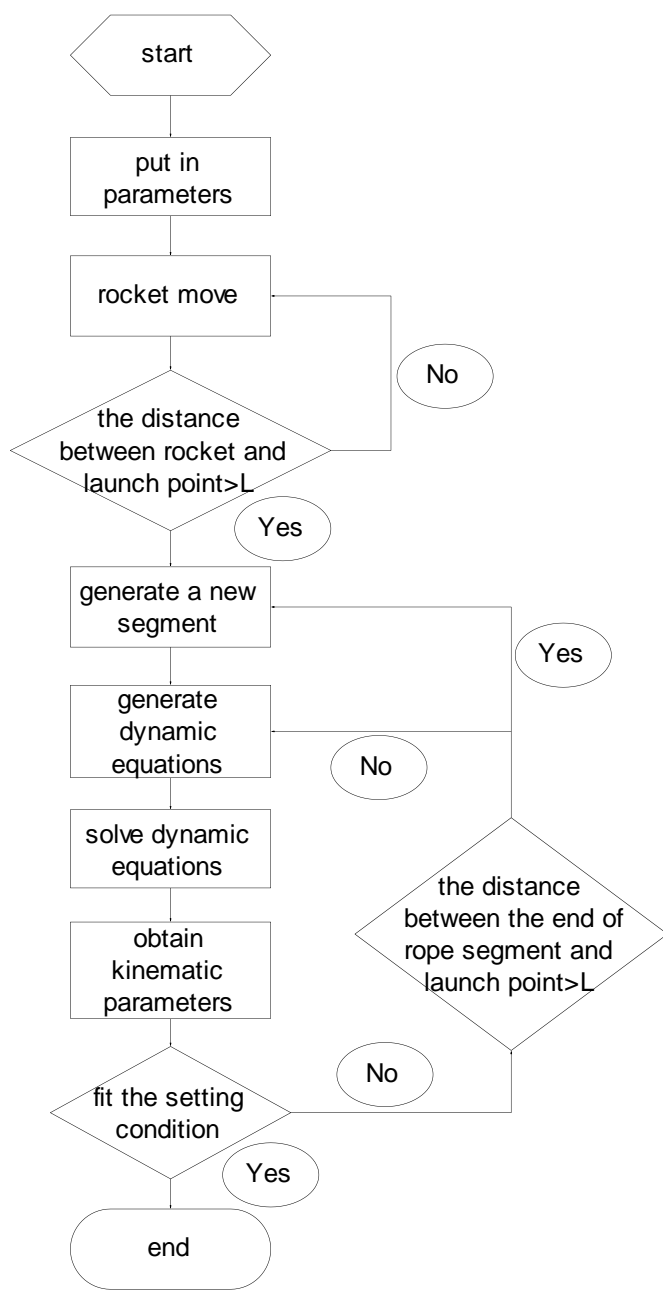

Fig. 2 Flow chart of the calculation

\section{Simulation calculation and discussion}

\subsection{Physical model}

Taking a rocket for example, where the rocket length is $1 \mathrm{~m}$, diameter $122 \mathrm{~mm}$, total weight $20 \mathrm{Kg}$, gunpowder weight $2.33 \mathrm{Kg}$, rocket total impulse is $4770 \mathrm{~N} \mathrm{~s}$, the engine working time $0.43 \mathrm{~s}$, linear density of the rope
$0.43 \mathrm{Kg} / \mathrm{m}$, each rope section is taken as $1 \mathrm{~m}$, emission angles $25^{\circ}, 35^{\circ}, 45^{\circ}, 55^{\circ}$.

\subsection{Law of motion}

1. The variation rule of the rocket trajectory when the launch angle changed from $25^{\circ}$ to $65^{\circ}$ is shown in Fig. 3. As can be seen from the chart, the trajectory is significantly asymmetric. The descending arc is steeper than the upward arc. The vertex distance is much larger than the half range. With the angle's increasing, top height of the trajectory increased. And the range increased at the beginning, when the angle reaches a certain value, the range decreases gradually. Under the same condition, there is an angle for the line throwing rocket to reach the maximum range.

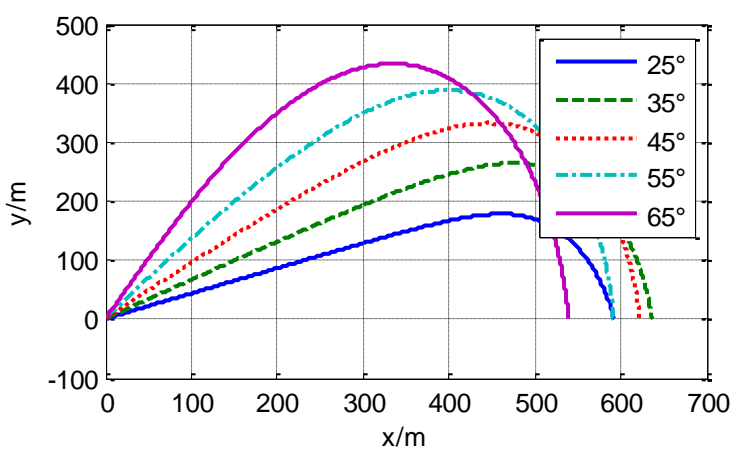

Fig. 3 The trajectory of different launch angles

2. The variation rule of the rocket velocity when the launch angle changed from $25^{\circ}$ to $65^{\circ}$ is shown in Fig. 4.

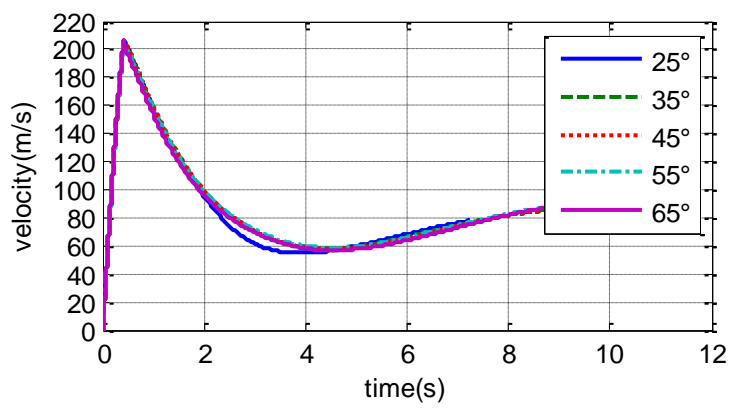

Fig. 4 The velocity of different launch angles

As can be seen from the chart, the velocity increased in a very short period of time from 0 to the maximum, and then began to decrease, reducing to a minimum, and then began to increase. In the boost phase, rocket engine worked, the thrust is larger than the air resistance, gravity of the rocket, the pulled rope and the force acting on end of the rope, so the velocity of the rocket increased rapidly until it reached the maximum value. When the rocket engine stopped work, the boost phrase ended, and the rocket kept rising with the act of inertia until it reached trajectory vertex. At the same time, the velocity began to decrease, being influenced by the air resistance, gravity and rope pulling force. Then the rocket changed the direction of movement and fly downward, and the velocity reduced to a minimum value. Gravity accelerated this movement trend, so the velocity of the rocket began to increase until it landed. With the angle increases, the time of the rocket flying in the air increases, and landing velocity of the rocket also increased. 
3. The variation rule of the rocket trajectory angle when the launch angle changed from $25^{\circ}$ to $65^{\circ}$ is shown in Fig. 5. As can be seen from the chart, at the beginning, trajectory angle changes slowly. Then it decreased rapidly from a positive to a negative value with the rocket flying. It kept decreasing until landed. It is because in the early stage of the rocket engine, thrust play the leading role, trajectory angle changes slowly, after the engine working, gravity plays the leading role, forcing the rocket to head down. And the rocket was at trajectory corresponding vertex when the trajectory angle equals 0 . The rocket landing angle was relatively large and the trajectory was relatively steep when the angle changes from $25^{\circ}$ to $65^{\circ}$.

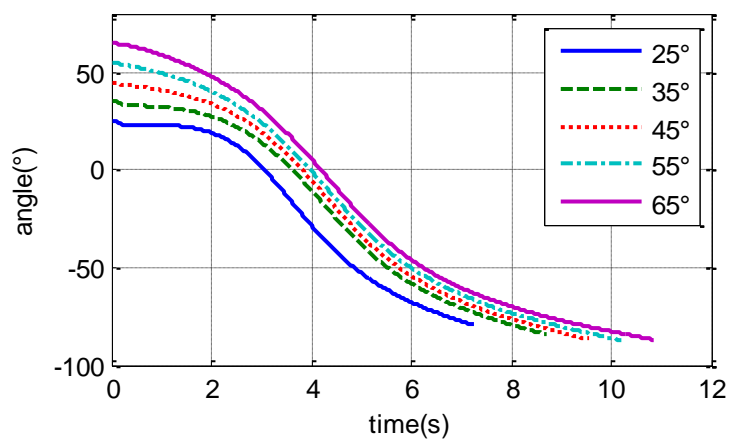

Fig. 5 Trajectory angle of different launch angles

4. The states of the rope in the air at different times are shown in Figure 6 while the launch angle is $45^{\circ}$. You can see: at the beginning, the rope is relatively straight, and latter, the middle part of the rope appears upwardly convex shape. It is mainly because during the early flight, the rocket has large engine thrust and the velocity is fast, the movement is basically along a straight line with the rope pulled out quickly, therefore, the rope is relatively flat. By the end of the flight, the rocket velocity has decreased, the motion of the rope back has lagged behind the front, while due to the effect of air resistance, and the middle part of the rope forms the upwardly convex sharp, which is in accordance with the actual situation.

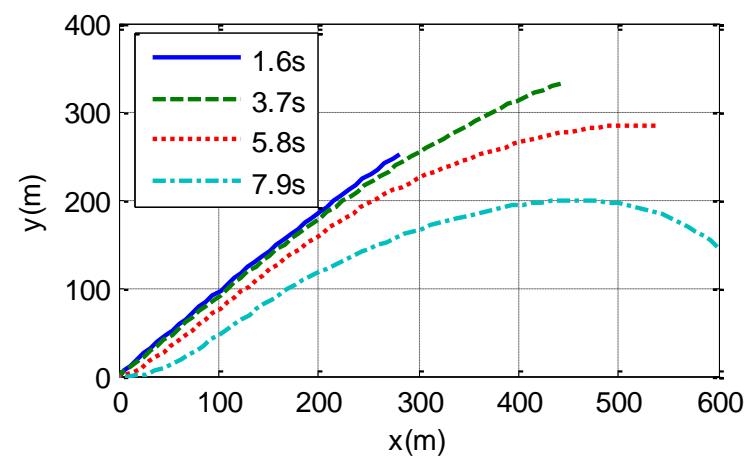

Fig. 6 Aviation gesture at different times

5. The acceleration curve is shown in Fig. 7 when the launch angle is $45^{\circ}$. You can see the acceleration at the initial time is positive, and the value is larger, then it begins to decrease. It mutated to negative at $0.43 \mathrm{~s}$ and continues to decrease. After it reduces to a minimum, it increased and crossed zero and reached a positive value, then the curve is relatively flat. The acceleration at the beginning was large because the engine thrust played a major role, as the velocity increases and segments of the flying rope increase, the air resistance and tension acting on the rope and rocket increase correspondingly. Hence, the rocket acceleration begins to decrease. Acceleration mutated because there is no thrust after the engine stopped work, and the air resistance and tension of the rope are in the opposite direction with the movement of rocket. The velocity of the rocket is at minimum when the acceleration is zero.

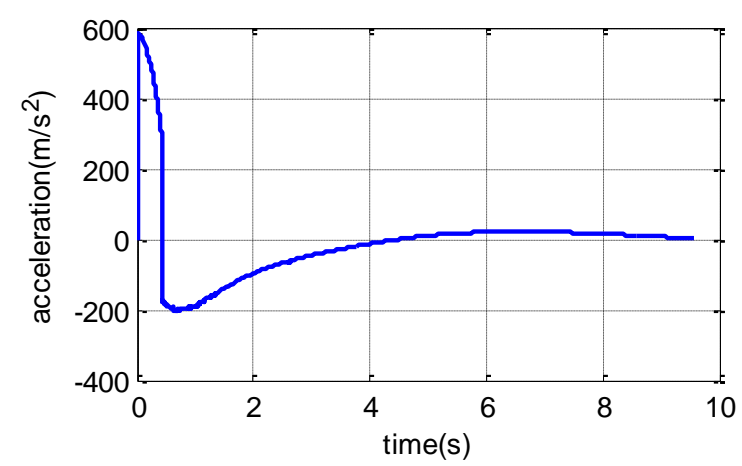

Fig. 7 The Acceleration of the Rocket

4.3. Comparison between Simulation Results and Experiment Results

According to the simulation parameters used in calculation, a certain type of rocket was tested. The test set is shown as Fig. 8:

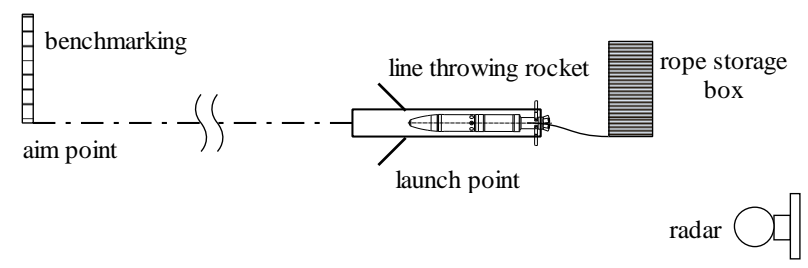

a

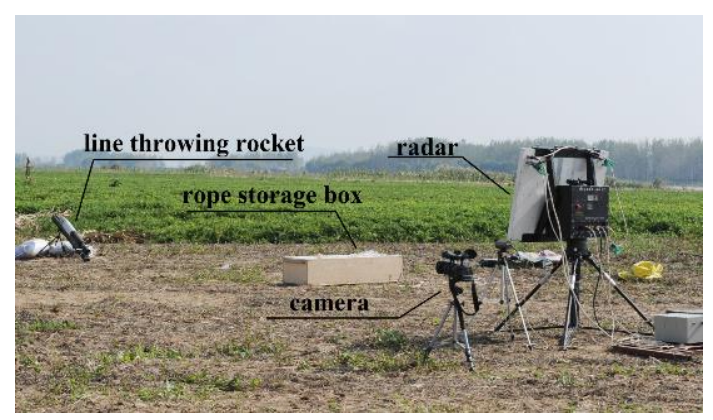

b

Fig. 8 a) The schematic diagram of the set; b) Set of the experiment

Data comparison between simulation results and experiment results can be seen in Table 3 and Table 4 . The range, maximum velocity, and the flight time obtained by simulation and test at the launch angle of $30^{\circ}$ are shown in Table 3. As can be seen from Table 3: the range measured in the simulation experiment is $7.2 \mathrm{~m}$ over the test data, the relative error is $1.13 \%$; maximum velocity of the simulation calculation is $7.1 \mathrm{~m} / \mathrm{s}$ faster than the measured test data, and the relative error is $3.4 \%$; the flight time of simulation is $0.5 \mathrm{~s}$ less than the test data. 
Comparison between simulation and experiment results at the launch angle of $30^{\circ}$

\begin{tabular}{|c|c|c|c|}
\hline Parameters & Range, $\mathrm{m}$ & Maximum velocity, $\mathrm{m} / \mathrm{s}$ & Flight time, $\mathrm{s}$ \\
\hline Simulation results & 637.1 & 206.6 & 8.1 \\
\hline Test results & 629.9 & 199.5 & 8.6 \\
\hline Absolute error & 7.2 & 7.1 & 0.5 \\
\hline
\end{tabular}

The range, maximum velocity and flight time obtained by simulation and test at the launch angle of $40^{\circ}$ are shown in Table 4. As can be seen from Table 4, the range of simulation is $10.1 \mathrm{~m}$ over the test data, the relative error is
$1.6 \%$; maximum velocity of the simulation calculation is $7.2 \mathrm{~m} / \mathrm{s}$ faster than the measured test data and the relative error is $3.5 \%$; flight time in the simulation is $0.6 \mathrm{~s}$ less than the test data.

Comparison between simulation and experiment results at the launch angle of $40^{\circ}$

\begin{tabular}{|c|c|c|c|}
\hline Parameters & Range, $\mathrm{m}$ & Maximum velocity, $\mathrm{m} / \mathrm{s}$ & Flight time, $\mathrm{s}$ \\
\hline Simulation results & 631 & 206.1 & 9.1 \\
\hline Test results & 620.9 & 198.9 & 9.7 \\
\hline Absolute error & 10.1 & 7.2 & 0.6 \\
\hline
\end{tabular}

The velocities of the rocket measured by radar and obtained by simulation at the launch angle of $30^{\circ}$ are shown in Fig. 9. From Fig. 9 we can see: the maximum velocity measured by the test is $199.5 \mathrm{~m} / \mathrm{s}$, the calculation maximum velocity is $206.6 \mathrm{~m} / \mathrm{s}$, after the engine stopped working; at $1 \mathrm{~s}, 2 \mathrm{~s}, 3 \mathrm{~s}$ and $4 \mathrm{~s}$, the test velocities measured were $154.3 \mathrm{~m} / \mathrm{s}, 99.3 \mathrm{~m} / \mathrm{s}, 73.8 \mathrm{~m} / \mathrm{s}$ and $60.5 \mathrm{~m} / \mathrm{s}$, and the calculation velocities are $158.7 \mathrm{~m} / \mathrm{s}, 98.9 \mathrm{~m} / \mathrm{s}, 70.3 \mathrm{~m} / \mathrm{s}$ and $58.5 \mathrm{~m} / \mathrm{s}$. The absolute errors are $2.8 \%, 0.4 \%, 5.0 \%$ and $3.4 \%$ with the average $2.9 \%$.

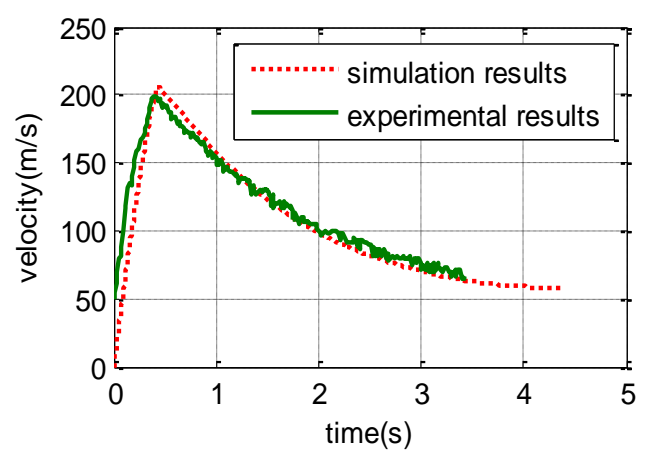

Fig. 9 Simulation and experiment Velocity Curve at the launch angle of $30^{\circ}$

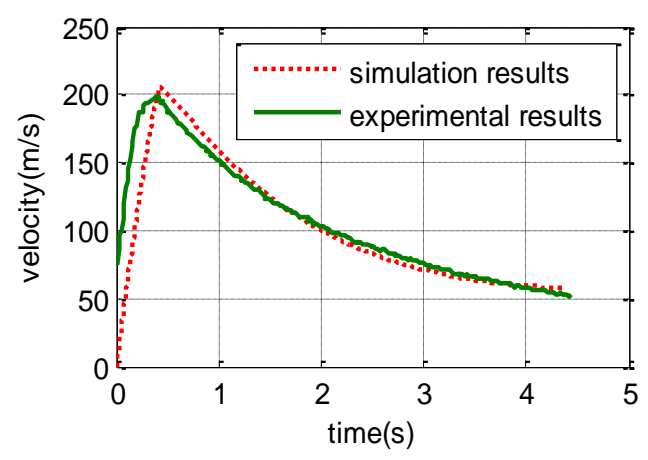

Fig. 10 Simulation and experiment Velocity Curve at the launch angle of $40^{\circ}$

The velocities of the rocket measured by radar and obtained by simulation at the launch angle of $40^{\circ}$ are shown in Fig. 10. From Fig. 10 we can see: the maximum velocity measured by the test is $198.9 \mathrm{~m} / \mathrm{s}$, the calculation maximum velocity is $206.1 \mathrm{~m} / \mathrm{s}$, after the engine stopped work; at $1 \mathrm{~s}$, $2 \mathrm{~s}, 3 \mathrm{~s}$ and $4 \mathrm{~s}$, the test velocities measured were $151.3 \mathrm{~m} / \mathrm{s}$, $103.2 \mathrm{~m} / \mathrm{s}, 76.1 \mathrm{~m} / \mathrm{s}$ and $58.1 \mathrm{~m} / \mathrm{s}$, and the calculation velocities are $159.9 \mathrm{~m} / \mathrm{s}, 100.5 \mathrm{~m} / \mathrm{s}, 71.4 \mathrm{~m} / \mathrm{s}$ and $59.4 \mathrm{~m} / \mathrm{s}$. The absolute errors are $5.4 \%, 2.7 \%, 6.6 \%$ and $2.2 \%$ with the average $4.2 \%$.

It can be seen from comparison of data in two tables and the velocity curves of two sets of test and simulations: the simulation results are very close to the experimental results and the velocity changes of the simulation and test are in good agreement, it also indicates that the dynamic model of the line throwing rocket is precise.

\section{Conclusions}

1. The line throwing rocket is analyzed through kinematics with the force acting on the rocket and the rope taken into account, which is transformed to the generalized active force and inertia force, and then the dynamic model of the flight of line throwing rocket was established.

2. The line throwing rocket model was simulated. And the calculation results show that: the dynamics model can simulate the movement of line throwing rocket effectively and reveal the law of its motion, thus, it is a feasible theoretic model.

3. The test results show that the dynamic model is relatively accurate.

4. The dynamic model is theoretically significant for the further study on the disturbance of the rope as well as guidance and flight control of the line throwing rocket.

\section{References}

1. McVey, D.F; Wolf, D.F. 1974. Analysis of deployment and inflation of large ribbon parachutes, Journal of Aircraft 11(2): 96-103. http://dx.doi.org/10.2514/3.60329.

2. Russell, J.J.; Anderson, W.J. 1977. Equilibrium and stability of a whirling rod-mass system, International Journal of Non-Linear Mechanics 12: 91-101. 
http://dx.doi.org/10.1016/0020-7462(77)90028-2.

3. Ablow, C.M.; Schechter, S. 1983. Numerical simulation of undersea cable dynamics, Ocean Engineering 10(6): 443-457. http://dx.doi.org/ 10.1016/0029-8018(83)90046-X.

4. Triantafyllou, M.S. 1985 . The dynamics of translating cables, Sound and Vibration 103(2): 171-182. http://dx.doi.org/10.1016/0022-460X(85)90231-7.

5. Niedzwecki, J.; Thampi, S.K. 1991. Snap loading of marine cable systems, Applied Ocean Research 13(5): 2-11. http://dx.doi.org/10.1016/S0141-1187(05)80045-8.

6. Kamman, J.W.; Huston, R.L. 1999. Modeling of variable length towed and tethered cable systems, Journal of Guidance, Control, and Dynamics 22(4): 602-608. http://dx.doi.org/10.2514/2.4423.

7. Driscoll, F.R.; Lueck, R.G.; Nabon, M. 1999. Development and validation of a lumped-mass dynamics model of a deep--sea ROV system, Applied Ocean Research 22: 169-182. http://dx.doi.org/10.1016/S0141-1187(00)00002-X.

8. Buckham, B.; Nahon, M. 1999. Dynamics simulation of low tension tethers, Oceans Conference Record(IEEE), Seattle, WA, USA, Institute of Electrical and Electronics Engineers Inc., Piscataway, NJ, USA: 757766. http://dx.doi.org/10.1109/OCEANS.1999.804904.

9. Buckham, B.; Nahon, M.; Cote, G. 2000. Validation of a finite element model for slack ROV tethers, Oceans Conference Record(IEEE), Providence, RI, USA, Institute of Electrical and Electronics Engineers Inc., Piscataway, NJ, USA: 1129-1136.

10. Johnny, E.; Quisenberry; Andrew S.; Arena. 2004. Dynamic simulation of low altitude aerial tow systems, AIAA Atmospheric Flight Mechanics Conference and Exhibit 16-19 August, Providence, Rhode Island. http://dx.doi.org/10.2514/6.2004-4813.

11. Choo, Y.; Casarella, M.J. 1973. A survey of analytical methods for dynamic simulation of cable-body systems, Journal of hydrometrics 7(4): 137-144. http://dx.doi.org/10.2514/3.62948.

12. Buckham, B.; Driscoll, F.R.; Meyer, N. 2004. Development of a finite element cable model for use in lowtension dynamic simulation, Journal of Applied Mechanics 71(4): 476-485. http://dx.doi.org/10.1115/1.1755691.

13. Buckham, B.; Driscoll, F.R. 2003. Three dimensional dynamics simulation of slack tether motion in an ROV system, Proceedings of the International Offshore and Polar Engineering Conference, Honolulu, Hawaii, United States, International Society of Offshore and Polar Engineers: 127-134.

14. Russell, J.J.; Anderson, W.J. 1977. Equilibrium and stability of a circularly towed cable subject to aerodynamic drag, Journal of Aircraft 14(7): 680-686. http://dx.doi.org/10.2514/3.58840.

15. Narkis, Y. 1978. Deployment forces in towing systems, Journal of Aircraft 15(2): 123-124. http://dx.doi.org/10.2514/3.58325.

16. Triantafyllou, M.S; Howell, C.T. 1993. Non-linear unstable response of hanging chains, Sound and Vibration 162(2): 263-280. http://dx.doi.org/10.1006/jsvi.1993.1118.
17. Triantafyllou, M.S; Howell, C.T. 1994. Dynamics response of cables under negative tension: an ill-posed problem, Sound and Vibration 173(4): 433-447. http://dx.doi.org/10.1006/jsvi.1994.1239.

18. Coomer, J.; Lazarus, M.; Tucker, R.W.; Kershaw, D.; Tegman, A. 2001. A non-linear eigenvalue problem associated with inextensible whirling strings, Journal of Sound and Vibration 239(5): 969-982. http://dx.doi.org/10.1006/jsvi.2000.3190.

19. Poole, L.R; Whitesids, J.L. 1974. Suspension-line wave motion during the lines-first parachute unfurling process, AIAA Journal 12(1): 38-43. http://arc.aiaa.org/doi/pdf/10.2514/3.49150.

20. Zhang Qingbin; Peng Yong; Chen Wenke et al. 2003. A mass-spring model with damping to the simulate the snatch process of parachte, Journal of Ballistics 15(1): 31-36.

http://dx.doi.org/10.3969/j.issn.1004499X.2003.01.007.

21. Wang Haitao; Qin Zizeng; Song Xu-min; Guo Peng; Tao Wusha. 2010. Analysis of the phenomenon of bull whipping in the deployment process of large parachute, Journal of National University of Defense Technology 32(5): 34-38. http://dx.doi.org/10.3969/j.issn.1001-2486.2010.05.007

22. Wu Xiaoping; Zheng Youxiang; Qiu Guangshen. 1995. The study on ballistics calculation of line throwing rocket, Journal of Ballistics (1): 41-49. http://www.cqvip.com/QK/92261X/199501/1708060.ht $\mathrm{ml}$.

23. Wu Hong; Li Kaiming 2003. Study on the air resistance characteristics in explosive hose of rocket-propelled line charge demolition device, Engineer Equipment Research (6): 42-44.

24. Wu Hong; Wang Yong 2006. The study of ballistic calculation in finite particles model of flexible body, Journal of Projectiles Rockets Missiles and Guidance 26(3): 162-165

http://dx.doi.org/10.3969/j.issn.1673-9728.2006.03.054

25. Huston, R.L.; Kamman, J.W. 1982. Validation of finite segment cable models, Computers and Structures 15(6): 653-660. http://dx.doi.org/10.1016/S0045-7949(82)80006-0.

26. Wang, Y.; Huston, R.L. 1994. A lumped parameter method in the nonlinear analysis of flexible multibody systems, Computers and Structures 50(3): 421-432. http://dx.doi.org/10.1016/0045-7949(94)90011-6.

27. Huston, R.L; Liu, Y.W. 1987. Dynamics of multibody systems, Tianjin: Tianjin University Publishing House.

28. Kamman, J.W.; Huston, R.L. 1985. Modeling of Submerged Cable Dynamics, Computers and Structures 20(103): 623-629. http://dx.doi.org/10.1016/0045-7949(85)90110-5.

29. Han Zipeng 2008. Ballistics of projectile and rocket, Beijing: Beijing Institute of Technology Press.

30. Dean Wolf 1971. Dynamics stability of a nonrigid Parachute and Payload system, Journal of Aircraft 8(8): 603-609.

http://dx.doi.org/10.2514/3.59145. 
Lu Ming, Gu Wenbin, Liu Jianqing, Wang Zhenxiong, $\mathrm{Xu}$ Jinling

DYNAMIC MODELING OF THE LINE THROWING ROCKET WITH FLIGHT MOTION BASED ON KANE'S METHOD

\section{S u m m a r y}

A dynamic model of the line throwing rocket flight is presented based on Kane's method. Through kinematic description of the line throwing rocket's flight, the rope is divided into $\mathrm{N}$ discrete finite segments by finite segment method. The dynamic model for the line throwing rocket is developed with the consideration of such forces as the gravity, thrust, aerodynamic forces and the tension in the rope during rocket flying. The numerical example shows that the numerical results are exactly consistent with experiments results and the numerical model of the line throwing rocket flight can be realized, revealing the motion law of the dynamic model. The dynamic model is a key theoretical support to the research on the disturbance of the rope, the guidance and flight control of the line throwing rocket.

Keywords: line throwing rocket; Kane's method; dynamic model; finite segment.

Received October 12, 2015

Accepted September 28, 2016 